\title{
Volume Reconstruction by Inverse Interpolation: Application to Interleaved MR Motion Correction
}

\author{
Torsten Rohlfing, Martin H. Rademacher, and Adolf Pfefferbaum \\ Neuroscience Program, SRI International, Menlo Park, CA, USA \\ torsten@synapse.sri.com
}

\begin{abstract}
We introduce in this work a novel algorithm for volume reconstruction from data acquired on an irregular grid, e.g., from multiple co-registered images. The algorithm, which is based on an inverse interpolation formalism, is superior to other methods in particular when the input images have lower spatial resolution than the reconstructed image. Local intensity bounds are enforced by an L-BFGSB optimizer, regularize the reconstruction problem, and preserve the intensity distribution of the input images. We demonstrate the usefulness of our method by applying it to retrospective motion correction in interleaved MR images.
\end{abstract}

\section{Introduction}

The fundamental problem addressed in this paper is the reconstruction of a volumetric image from data acquired on an irregular grid. This also includes data acquired on multiple regular grids, e.g., multiple images with general coordinate transformations between them. Such image reconstruction problems arise, for example, in freehand threedimensional (3D) ultrasound acquisition [1] or in the reconstruction of high-resolution motion-corrected magnetic resonance (MR) images [4].

A common and straightforward volume reconstruction method, pursued for example by Moore et al. [2] in the construction of a cardiac MR atlas, is to interpolate the coregistered acquired images onto the target grid and average the interpolated images. This approach is limited, however, by the resolution of the input images (Fig. 1). It is, therefore, not satisfactory for reconstruction of high-resolution images from lowresolution images, as it is encountered in spatially interleaved image acquisitions. Such acquisitions are used in MR imaging to minimize crosstalk between neighboring slices without introducing slice gaps, and for covering large volumes when acquiring images with repetition times (TR) too short to allow data from all slices to be collected in a single pass. After acquisition of multiple interleaved passes, these are combined to form a high-resolution 3D image volume.

Movement of the subject is particularly a problem in the very young, the very old and in those suffering from neurodegenerative conditions. Motion artifacts within a pass are not recoverable post reconstruction, whereas motion across passes leads to misalignment of the images in the separate passes relative to each other. This, in turn, causes characteristic artifacts in the resulting image stacks (see Fig. 11 for an example), which makes them unsuitable for 3D image processing (e.g., registration, multi-spectral image segmentation). One application for the algorithm introduced in this paper is to 


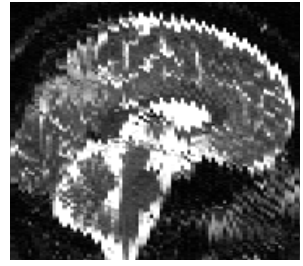

(a)

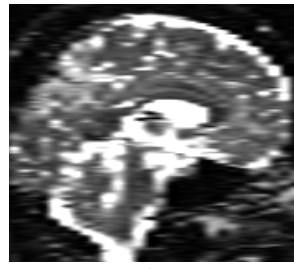

(b)

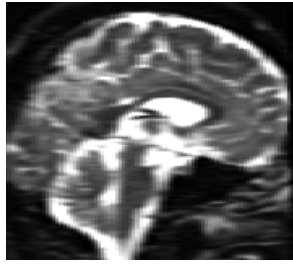

(c)

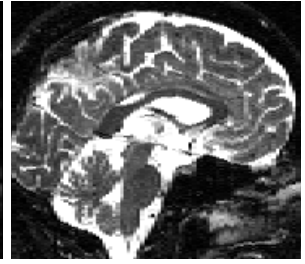

(d)

Fig. 1. Example of motion artifacts in a three-pass interleaved late-echo fast spin echo MR image (coronal slice orientation). The subject moved mostly between the first and second pass. (a) Characteristic artifacts are particularly evident in the sagittal view. (b) The pass images (here, pass \#0) have $1 / 3$ of the spatial resolution in between-slice direction (here, $6 \mathrm{~mm}$ instead of $2 \mathrm{~mm}$ ). (c) The average of co-registered pass images (analogous to Ref. [2]) is blurry. (d) The image reconstructed using the proposed algorithm is crisp and free of between-pass motion artifacts.

reconstruct high-resolution, motion-corrected images from co-registered low-resolution passes with arbitrary rigid transformations between them.

\section{Methods}

The method for retrospective reconstruction of pass-to-pass motion artifact corrected images proposed in this paper comprises three distinct stages, which are described in detail below: 1) using image-to-image registration, the parameters of subject motion between the passes are estimated; 2) an initial reconstructed image is generated by volume injection of the co-registered pass images into the reconstructed image grid; 3) the final artifact corrected image is computed in an iterative image reconstruction stage, which represents the primary contribution of this paper. The reconstruction, which in effect serves to unblur the initial, volume-injected image, is based on an inverse interpolation formalism, which is solved using a gradient descent minimization of the mean squared difference (MSD) between the actually acquired pass images and those interpolated from the evolving artifact-corrected image.

\subsection{Motion Estimation by Image Registration}

The images acquired in the different passes are first combined into separate, contiguous 3D image stacks (rather than a single, interleaved high-resolution stack). To recover the motion between the passes, one of them (for example the first) is selected as the reference, and the images from the remaining passes are registered to the reference pass image using an established intensity-based 3D rigid registration algorithm [3], but using MSD as a more appropriate registration criterion in this application.

The transformation that relates the coordinate system of the $n$-th pass to the reference coordinate system is denoted as $\mathbf{T}_{n}$. Note that the we can restrict consideration to rigid coordinate transformations in this paper as dictated by the application, but this is not a limitation of the volume reconstruction method. Of course, estimating more complex nonrigid motion between low-resolution images is increasingly complicated 
and under-constrained, and registration errors will propagate to, and cause error in, the reconstruction stage.

\subsection{Volume Injection}

Image reconstruction from multiple co-registered views can be solved by push-forward interpolation and averaging [4]: Each pixel $\vec{y}$ is injected into the reconstructed image volume at all locations $\vec{x}$ within a radius $d$, weighted using a truncated Gaussian-shaped kernel,

$$
G_{\vec{y}, \sigma}(\vec{x}) \propto \begin{cases}\exp \left(-\frac{(\vec{x}-\vec{y})^{2}}{2 \sigma^{2}}\right) & \text { if }\|\vec{x}-\vec{y}\|_{2} \leq d, \\ 0 & \text { otherwise. }\end{cases}
$$

The accumulated intensities at each reconstructed pixel are subsequently normalized with the sum of all weights that contributed to them, so as to account for different numbers and distances of contributing pixels.

\subsection{Volume Reconstruction by Inverse Interpolation}

We now cast the actual image reconstruction of the high-resolution motion corrected 3D image stack from the co-registered passes as an inverse interpolation problem. Ignoring the spatial arrangement of its pixels, we can denote the (unknown) ground truth image as a vector of pixel values, $\vec{f} \in \mathbb{R}^{M}$, where $M$ is the number of image pixels. The images from the separate passes are denoted $\vec{u}^{(n)} \in \mathbb{R}^{M_{n}}$ where $n$ is the index of the pass, and $M_{n}$ is the number of pixels acquired in the respective pass.

For the purpose of image reconstruction, the acquired pass images are modeled as if they were interpolated from $\vec{f}$. Each such interpolation can be written as a matrix-vector multiplication

$$
\vec{u}^{(n)}=\mathbf{W}^{(n)} \vec{f},
$$

where $\mathbf{W}^{(n)} \in \mathbb{R}^{M_{n} \times M}$ is the matrix of interpolation coefficients for the $n$-th pass. The elements of each $\mathbf{W}^{(n)}$ essentially describe for each interpolated image pixel, which pixels in the original image contribute to it with what interpolation weight. The matrix entries are, therefore, determined by a) the spatial arrangements of the pixels in the images, b) the coordinate transformations between the images, and c) the interpolation kernel (e.g., linear, cubic, sinc).

The inverse interpolation problem is to find the image $\vec{f}$ that satisfies the system of equations defined by Eq. (2) over all $n$. Due to the size of the interpolation matrices, incomplete and inconsistent data, this system cannot be solved directly. Instead, we devised an iterative least-squares approach, for which we let $\vec{v}$ be some approximation of $\vec{f}$. Then the least-squares approximation error can be written as

$$
E(\vec{v})=\sum_{n}\left(\vec{u}^{(n)}-\mathbf{W}^{(n)} \vec{v}\right)^{2}
$$

and the true solution is approximated by solving the following minimization problem:

$$
\vec{f} \approx \arg \min _{\vec{v}} E(\vec{v}) .
$$


The analytical gradient of $E$ with respect to the elements of $\vec{v}$ (i.e., the pixel intensities in the evolving reconstructed image) is derived using elementary calculus as follows:

$$
\begin{array}{r}
\frac{\partial}{\partial v_{i}} E(\vec{v})=\sum_{n} \frac{\partial}{\partial v_{i}}\left(\vec{u}^{(n)}-\mathbf{W}^{(n)} \vec{v}\right)^{2}=\sum_{n} \sum_{j} \frac{\partial}{\partial v_{i}}\left(u_{j}^{(n)}-\sum_{k} \mathbf{W}_{j, k}^{(n)} v_{i}\right)^{2} \\
=-2 \sum_{n} \sum_{j}\left[\mathbf{W}_{j, i}^{(n)}\left(u_{j}^{(n)}-\sum_{k} \mathbf{W}_{j, k}^{(n)} v_{k}\right)\right]
\end{array}
$$

Substituting into this equation for each pass $n$ the error image, $\vec{e}^{(n)}=\vec{u}^{(n)}-\mathbf{W}^{(n)} \vec{v}$,

$$
\frac{\partial}{\partial v_{i}} E(\vec{v})=-2 \sum_{n} \sum_{j}\left[\mathbf{W}_{j, i}^{(n)} \vec{e}_{j}^{(n)}\right] .
$$

Note that $E$ is non-negative, and zero if and only if the acquired pass images are identical to those interpolated in their spatial position from the reconstructed image, i.e., when $\vec{u}^{(n)}=\mathbf{W}^{(n)} \vec{v}$ for all $n$. Note also that if coordinate transformation $\mathbf{T}_{n}$ is the identity transformation, then for the common interpolation kernels the corresponding interpolation matrix $\mathbf{W}^{(n)}$ is the identity matrix. Therefore, if $\mathbf{T}_{n} \equiv \operatorname{Id}$ for all $n$ (i.e., if there is no motion between the passes), then the acquired image itself is the global optimum of the reconstruction error functional in Eq. (3).

\subsection{Implementation of the Inverse Interpolation}

To compute the gradient of $E$, the inner sum in Eq. (6) must be evaluated for all $j$ for which $\mathbf{W}_{j, i}^{(n)} \neq 0$, i.e., for all $i, j, n$ such that the $j$-th pixel in the $n$-th interpolated pass image depends on the $i$-th pixel in the reconstructed image. The relationships between the pixels in reconstructed and interpolated images are schematically illustrated in Fig. 2. For forward interpolation, the interpolated image pixel is mapped into the reconstructed image via transformation $\mathbf{T}$ and its value determined from the neighboring reconstructed pixels.

The inverse interpolation determines the pixels in the interpolated image which depend on a given pixel in the reconstructed image. When the reconstructed pixel's interpolation neighborhood is mapped into the interpolated image via the inverse transformation, $\mathbf{T}^{-1}$, these are all interpolated image pixels that are located inside the mapped region. For affine (or rigid) transformations, an elegant and efficient way to compute the interpolated pixels inside the mapped neighborhood is via a volume intersection algorithm [5]. However, it is much easier (and almost as computationally efficient) to simply test for each pixel inside the bounding box (dashed rectangle in Fig. 22 (b)) of the mapped region corners whether or not transformation $\mathbf{T}$ maps it into that region.

\subsection{Local Intensity Thresholding}

The acquired MR images represent a dense, approximately uniform sampling of the imaged object. Consequently, the intensity distribution in the original images should be 

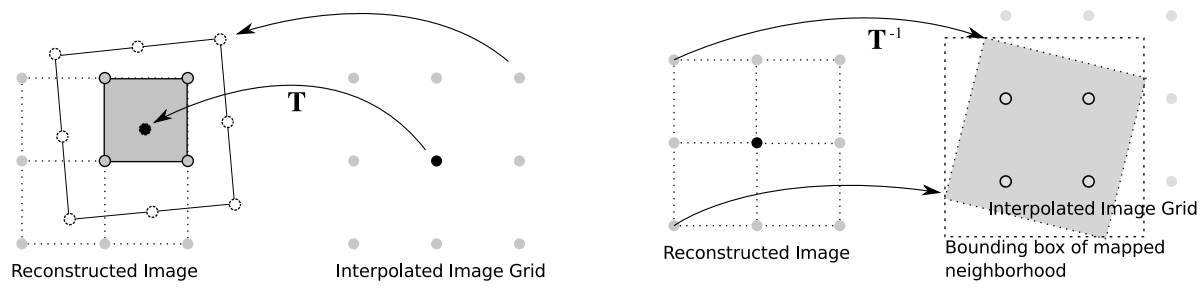

Fig. 2. Illustration of pixel relationships in forward (left) and inverse interpolation (right)
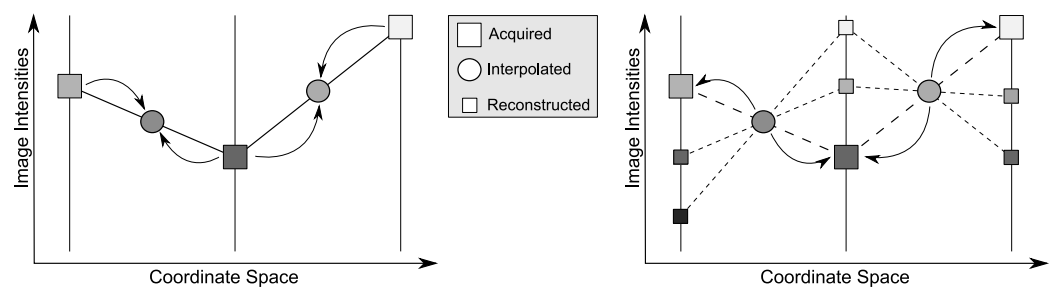

Fig. 3. Illustration of ill-posedness of inverse interpolation. Left: Given the acquired data (large squares), the forward problem of determining interpolated data (circles) between the grid positions is well-defined. Right: For the inverse problem to reconstruct the acquired data from the interpolated data, there is a continuum of solutions. Some examples of such solutions are represented here by smaller squares and dashed lines.

a good approximation of the "true" distribution for the given subject, and it is reasonable to expect that the reconstructed image should preserve this distribution. This is, however, not the case, largely due to the fact that inverse interpolation is an ill-posed problem for which there is no unique solution. This is schematically illustrated in Fig. 3 Meaningful boundary conditions are also not available in general, because at least one face of human MR image volumes is always a truncation surface.

A common remedy for this type of problem is to add a regularization term to the optimized cost function (here, the difference between interpolated and acquired pass images) that favors solutions which possess additional properties. For example, one could use the Kullback-Leibler (KL) divergence [6] between the acquired and the reconstructed image intensity distributions. Solutions would thus favor reconstructed images that have the same intensity distribution as the acquired images. However, we found such an approach difficult to implement due to the need to compute gradients of the KL divergence with respect to the reconstructed image intensities. It is also potentially too strict and may prevent effective optimization.

Instead, we apply a simple local thresholding scheme that makes use of the assumption that the intensity of any reconstructed pixel should be inside the range of pixel values in the original images that contribute to this pixel. In other words, the acquired intensity range in a reconstructed pixel's local neighborhood constrains the permissible intensities for that pixel. Our implementation enforces this constraint by hard truncation, rather than by soft penalty terms. This is more computationally efficient, 
completely avoids smoothing of the reconstructed image, and is easily enforced during the minimization of $E$ using an L-BFGS-B optimizer [7].

\section{Results}

In the absence of a gold standard, we judge the reconstructed images by two criteria: 1) visual assessment of artifact reduction and definition of anatomical detail, and 2) preservation of acquired image intensity distribution. For visual assessment, Fig. 4 shows original and motion-corrected images from eight subjects. Inverse interpolation used cubic interpolation and local intensity thresholding. Note the visible increased crispness of the reconstructed images and the substantially reduced approximation error, $E$, after inverse interpolation compared with those after volume injection.

For one of the images in Fig. 4, the histogram plots in Fig. 5 show the intensity distributions of artifact-corrected images, reconstructed using three different interpolation kernels and with and without local thresholding. For comparison, the distribution of the original image data is also shown in each plot. For each combination, the final MSD
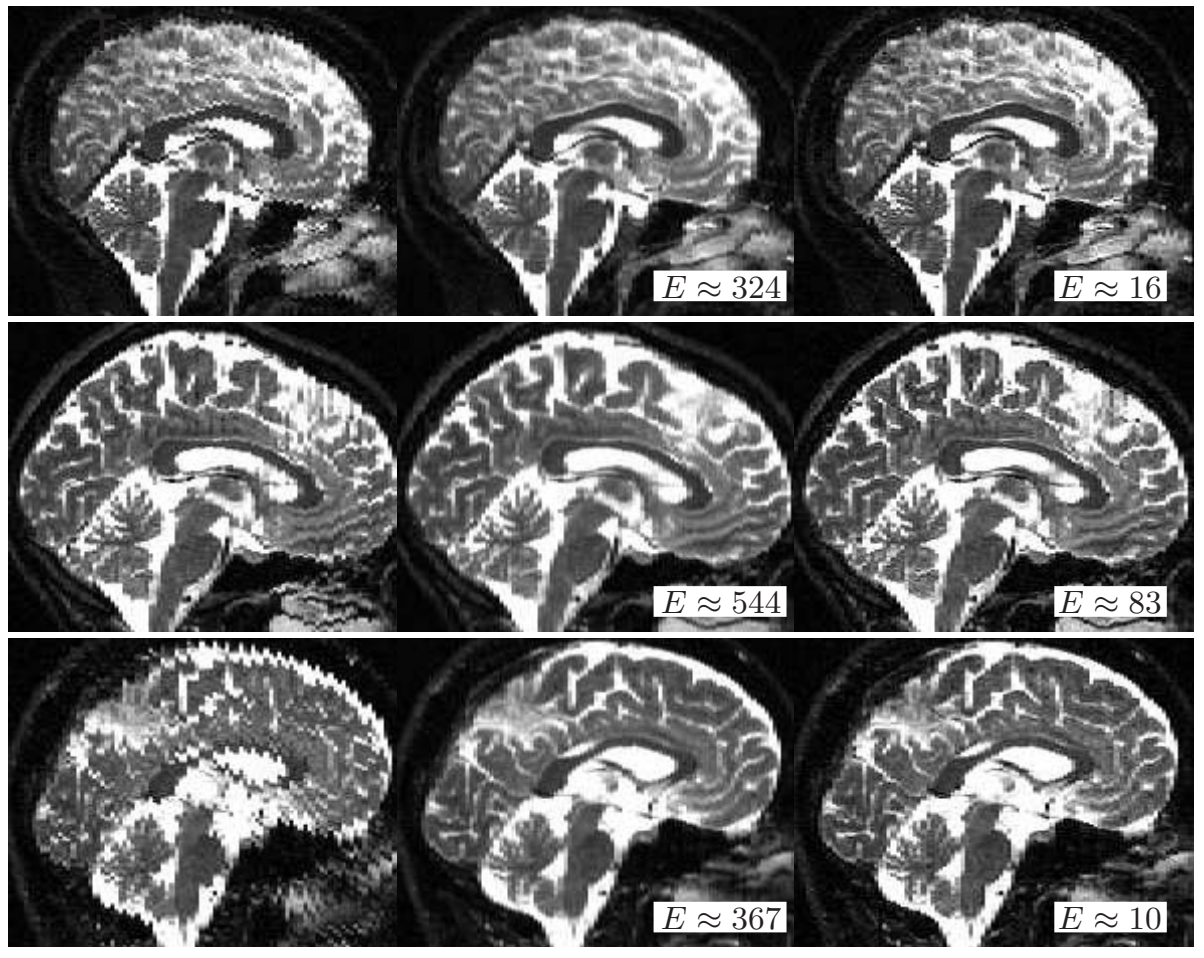

Fig. 4. Reconstructed motion-corrected images (volume injection and inverse interpolation) from three subjects. Image acquisition used a coronal fast spin echo sequence (94 slices $2 \mathrm{~mm}$ thick; $\mathrm{TR} / \mathrm{TE}=11050 / 98 \mathrm{~ms}$, matrix $=256 \times 192, \mathrm{FOV}=240 \mathrm{~mm}$ ), requiring three interleaved passes. For each reconstructed image, the approximation error, $E$, of the acquired images is provided. 

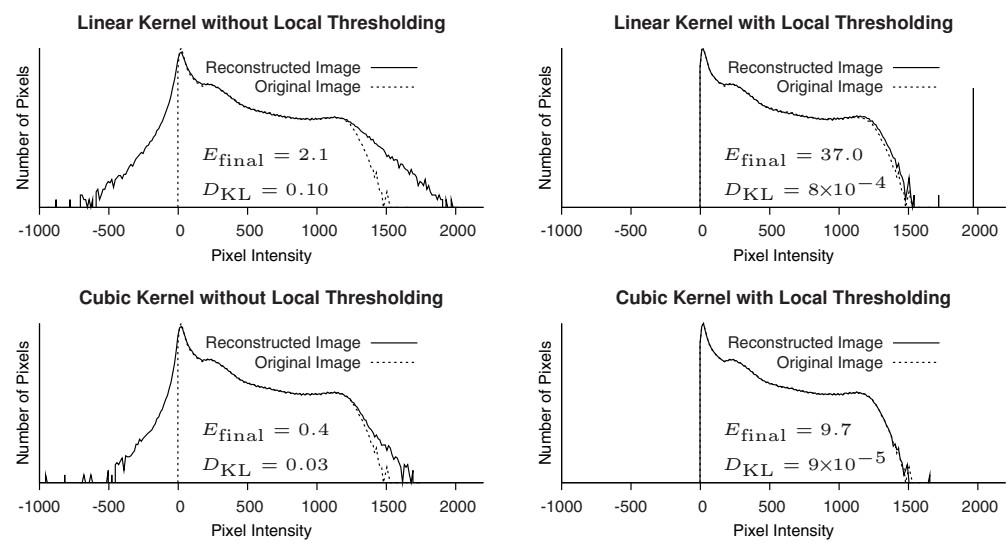

Sinc Kernel without Local Thresholding

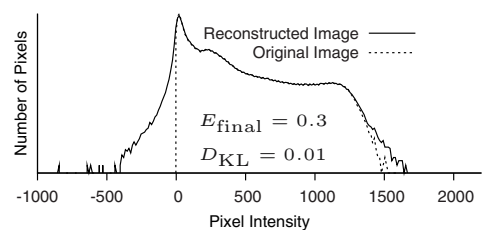

Sinc Kernel with Local Thresholding

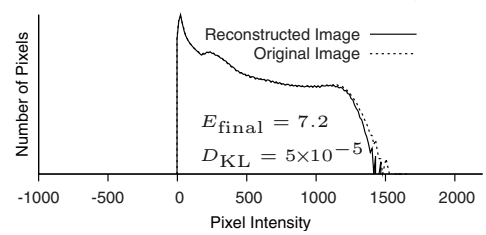

Fig. 5. Comparison of reconstructed vs. acquired image intensity distributions for different interpolation kernels, with and without local thresholding. Sinc interpolation used a cosine window with 3 samples window radius. All plots have logarithmic vertical axes. Note in particular the introduction of artificial high and low image intensities when local thresholding is not used. In each panel, $E_{\mathrm{final}}$ is the final MSD between interpolated and acquired pass images, and $D_{\mathrm{KL}}$ is the KL divergence between the original and reconstructed image intensity distributions.

Table 1. Computation times for evaluating the gradient $\frac{d}{d \vec{v}} E(\vec{v})$ and for a single evaluation of the approximation error (one forward interpolation). Times were measured on a dual-CPU Intel Xeon (E5345) server at $2.33 \mathrm{GHz}$ clock speed. Sinc interpolation used a cosine window with 3 samples window radius. Optimization using the L-BFGS-B algorithm [7] typically converges in under 20 gradient search iterations.

Computation Time: Single CPU (8 CPUs)

\begin{tabular}{rccc} 
& Linear & Cubic & Sinc \\
\hline Gradient Computation & $138 \mathrm{~s}(22 \mathrm{~s})$ & $354 \mathrm{~s}(55 \mathrm{~s})$ & $1964 \mathrm{~s}(262 \mathrm{~s})$ \\
Error Computation & $4 \mathrm{~s}(1 \mathrm{~s})$ & $9 \mathrm{~s}(2 \mathrm{~s})$ & $54 \mathrm{~s}(7 \mathrm{~s})$ \\
\hline
\end{tabular}

between interpolated and acquired images, $E_{\mathrm{final}}$, and the KL divergence, $D_{\mathrm{KL}}$, between the original and the reconstructed image intensity distributions are also given.

The results in Fig. 5 are typical for all images to which we applied our algorithm. Higher-order interpolation improves the approximation, and to some extent it also reduces the frequency of artificial values outside the original image intensity range. 
Local thresholding not only prevents values outside that range, which it is guaranteed to do by design, but also very effectively produces global intensity distributions that are virtually identical to the acquired image intensity distribution. This is remarkable because local thresholding does not explicitly enforce any global properties of the reconstructed image intensity distribution.

\section{Conclusion}

The algorithm introduced herein reconstructs high-resolution images from data acquired on an irregular grid, such as multiple co-registered lower resolution images. The coordinate transformations between the input images are arbitrary, i.e., the algorithm is not limited to in-plane transformations.

While this is by no means the only application of our method, we have demonstrated herein its usefulness for retrospective correction of between-pass motion in interleaved MR imaging. The qualitative (visual appearance) and quantitative (approximation error; preservation of intensity distribution) results in this paper suggest that a simple cubic kernel is effective, and it is also computationally efficient (Table 1). For comparison, we have demonstrated the superiority of our inverse interpolation reconstruction approach over interpolation-and-averaging schemes described in the literature [2]. It is also worth mentioning that our method can reconstruct image volumes from lower-dimensional acquired data (e.g., freehand ultrasound), which interpolation and averaging can not.

The advantage of our method over volume injection techniques [14] is more subtle, but still visually apparent (Fig. 4). Unlike volume injection, which requires tuning of injection kernel shape and truncation radius, our method is also data independent and parameter free. This is important because there may not be volume injection parameters that work equally well everywhere in the image, depending on the spatial arrangement of the acquired data.

\section{Acknowledgments}

This work was supported through Grants AA05965, AA12388, and AG17919. The authors thank Michael P. Hasak for many helpful comments and suggestions.

\section{References}

1. Prager, R.W., Gee, A., Berman, L.: Stradx: real-time acquisition and visualization of freehand three-dimensional ultrasound. Med. Image. Anal. 3(2), 129-140 (1999)

2. Moore, J., Drangova, M., Wierzbicki, M., Barron, J., Peters, T.: A high resolution dynamic heart model based on averaged MRI data. In: Ellis, R.E., Peters, T.M. (eds.) MICCAI 2003. LNCS, vol. 2878, pp. 549-555. Springer, Heidelberg (2003)

3. Studholme, C., Hill, D.L.G., Hawkes, D.J.: Automated three-dimensional registration of magnetic resonance and positron emission tomography brain images by multiresolution optimization of voxel similarity measures. Med. Phys. 24(1), 25-35 (1997) 
4. Rousseau, F., Glenn, O., Iordanova, B., Rodriguez-Carranza, C., Vigneron, D., Barkovich, J., Studholme, C.: A novel approach to high resolution fetal brain MR imaging. In: Duncan, J.S., Gerig, G. (eds.) MICCAI 2005. LNCS, vol. 3749, pp. 548-555. Springer, Heidelberg (2005)

5. Rohlfing, T.: Incremental method for computing the intersection of discretely sampled mdimensional images with n-dimensional boundaries. In: Medical Imaging: Image Processing, Proceedings of the SPIE, vol. 5032, pp. 1346-1354 (February 2003)

6. Kullback, S., Leibler, R.A.: On information and sufficiency. Ann. Math. Stat. 22, 79-86 (1951)

7. Byrd, R.H., Lu, P., Nocedal, J., Zhu, C.: A limited memory algorithm for bound constrained optimization. SIAM J. Sci. Comput. 16(5), 1190-1208 (1995) 